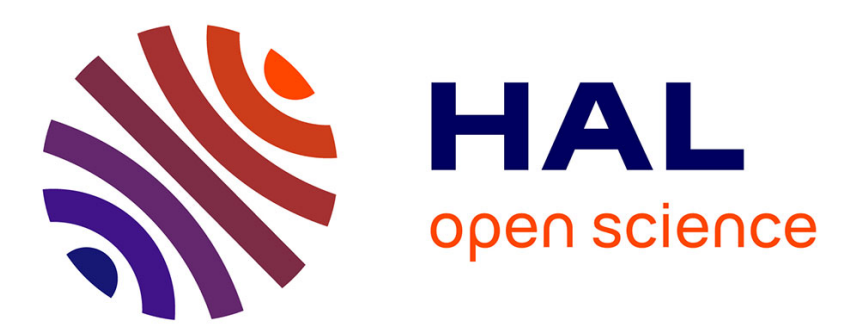

\title{
Contrôle hydrodynamique de l'organisation des biofilms en milieu eaux usées
}

Rim Al Khatib, Bruno Lartiges, Christian Mustin, Salah Skali-Lami

\section{To cite this version:}

Rim Al Khatib, Bruno Lartiges, Christian Mustin, Salah Skali-Lami. Contrôle hydrodynamique de l'organisation des biofilms en milieu eaux usées. La Houille Blanche - Revue internationale de l'eau, 2011, 5, pp.29-33. 10.1051/lhb/2011049 . hal-02424334

\section{HAL Id: hal-02424334 \\ https://hal.science/hal-02424334}

Submitted on 27 Dec 2019

HAL is a multi-disciplinary open access archive for the deposit and dissemination of scientific research documents, whether they are published or not. The documents may come from teaching and research institutions in France or abroad, or from public or private research centers.
L'archive ouverte pluridisciplinaire $\mathbf{H A L}$, est destinée au dépôt et à la diffusion de documents scientifiques de niveau recherche, publiés ou non, émanant des établissements d'enseignement et de recherche français ou étrangers, des laboratoires publics ou privés. 


\title{
CONTROLE HYDRODYNAMIQUE DE L'ORGANISATION DES BIOFILMS EN MILIEU EAUX USEES
}

\author{
R. Al Khatib ${ }^{1}$, B. Lartiges ${ }^{1}$, C. Mustin ${ }^{2}$, S. Skali-Lami ${ }^{3}$ \\ ${ }^{(1)}$ Nancy Université-INPL-LEM-ENSG/INPL-CNRS, Pôle de l'Eau, 15 avenue du Charmois, BP40, \\ 54501 Vandoeuvre Cedex, France, Tél : 33 (0)3 835962 97- Fax : 33 (0)3 83596255. \\ ${ }^{(2)}$ Nancy Université-INPL-LIMMOS-FST/UHP-CNRS 7137 boulevard des Aiguillettes, BP 239, 54506 \\ Vandoeuvre Cedex, France, Téléphone : +33 (0)3 836842 82- Fax : +33 (0)3 83684284. \\ ${ }^{(3)}$ École nationale supérieure d'électricité et de mécanique ENSEM-INPL, 2 avenue de la Forêt de Haye, \\ BP 160 - F-54504 Vandœuvre lès Nancy, Tél./phone +33 (0)3 83595552 - Fax : +33 (0)3 83595551.
}

E-mail: reem_el_khatib@yahoo.com

\begin{abstract}
Résumé La formation des biofilms sur les surfaces internes des réseaux d'assainissement à l'interface liquide-solide joue un rôle prépondérant dans la concentration des polluants, leur transport, leur dégradation, leur relargage et leur devenir dans le réseau. Le développement d'un biofilm est un phénomène complexe et influencé par plusieurs facteurs parmi lesquels les conditions hydrodynamiques sont les plus importantes. En effet, l'épaisseur, la quantité de biomasse fixée, la nature et la quantité d'EPS produits ainsi que la répartition des colonies bactériennes varient largement suivant le mode d'écoulement et de cisaillement appliqués. Ainsi le développement des biofilm des eaux usées sous écoulement Couette-Poiseuille sous deux vitesses de cisaillement n'est pas similaire dans les deux cas. Les observations par microscopie confocale, après marquage des biofilm par l'acridine orange, montrent que les biofilms obtenus à cisaillement $1 \mathrm{~s}^{-1}$ sont épais, peu denses, poreux et présentent des élongations (streamers) qui oscillent dans le flux alors que des biofilms générés en cisaillement $6 \mathrm{~s}^{-1}$ sont denses et peu épais. En appliquant une configuration d'écoulement avec un gradient pariétal nul le développement du biofilm se trouve freiné pour plus de deux jours. Cette configuration pourrait servir pour prévenir la formation de biofilm dans les différents systèmes.
\end{abstract}

Mots clé : Biofilm ; force de cisaillement ; exopolymères ; écoulement couette-poiseuille

\section{Introduction}

Les biofilms sont des associations de microcolonies bactériennes d'espèces différentes qui se développent sur toute surface à l'interface liquide-solide dans des conditions variées de température, apport en nutriments, conditions hydrodynamiques,... [1]. Dans les réseaux d'assainissement, la formation des biofilms joue un rôle primordial dans la transformation, le stockage et l'élimination des polluants. Les changements de conditions hydrodynamiques, le vieillissement et la quantité de substrat dans le milieu affectent les caractéristiques physicochimiques des biofilms et peuvent provoquer le détachement de ces derniers libérant ainsi les micropolluants dans le réseau [2]. Bien que la développement de biofilm soit un phénomène fréquent voire systématique à l'interface solide/liquide en présence de bactéries, sa formation passe par une séries d'étapes influencées par différents facteurs (mode d'écoulement, la nature de la surface, le type des nutriments et leurs concentrations, conditions d'aération). La formation du biofilm met donc en œuvre plusieurs processus physicochimiques (diffusion, adhésion, ...) ainsi que des processus biologiques (formation d'agrégats, croissance de microorganismes, production d'exopolymères, ...) $[3,4]$. Jusqu'à présent les différentes études réalisées sur le mécanisme de formation de biofilm ne peuvent pas présumer d'une séquence d'événements bien définie. 


\section{Influence des conditions hydrodynamiques}

L'activité du biofilm est fortement liée à son volume, la quantité de biomasse fixée, sa densité, sa porosité et le transfert de nutriments à l'intérieur du biofilm [5]. La vitesse d'écoulement du liquide et les forces de cisaillement qui en résultent semble être le facteur le plus important influençant la structure du biofilm vu leur impact sur la répartition des microcolonies et la production des exopolymères [6,7]. En effet, les différentes études menées sur la formation des biofilms à partir des systèmes naturels ou des souches pures sous différentes conditions hydrodynamiques montrent que l'épaisseur et la quantité de masse fixée, la nature et la quantité d'EPS produits ainsi que la répartition des colonies bactériennes varient largement suivant le mode de cisaillement appliqué [3, 7, 8]. Les écoulements à grande vitesse voire écoulement turbulent engendrent des biofilms denses et favorisent le transfert des nutriments depuis le liquide extérieur vers le biofilm ce qui favorise la production de exopolymères notamment les polysaccharides, ainsi le biofilm détermine une structure plus rigide et la cohésion entre les cellules sera plus importante [9]. Mais ceci aura un effet défavorable sur le transfert de nutriments au sein même du biofilm ce qui va diminuer l'activité de celui-ci et les biofilms seront plus fins $[3,10,11]$. A faible force de cisaillement, les biofilms sont beaucoup moins denses, plus poreux et plus épais. Ces biofilms sont formés par l'agrégation de colonies bactériennes séparées par des vides interstitiels qui créent une sorte de canalisations qui assurent le transfert de nutriments à l'intérieur du biofilm ce qui augmente son activité et aboutit à des biofilms plus épais mais moins rigides car la production des EPS est moins importante [3, 11].

Nous nous intéressons dans ce travail, à la croissance de biofilms en milieu eaux usées urbaines dans des conditions contrôlées d'écoulement et de cisaillement. Ainsi un réacteur Couette poiseuille a été choisi pour la réalisation des expériences et la croissance des biofilms à partir des eaux usées.

\section{Matériels et Méthodes}

\section{Réacteur de croissance}

Le réacteur Couette poiseuille (figure 1) est formé de deux cylindres concentriques en polychlorure de vinyle (PVC) espacé de $3,5 \mathrm{~mm}$. Le cylindre extérieur de rayon $5 \mathrm{~cm}$ et de hauteur $10 \mathrm{~cm}$ est statique et porte 16 coupons de prélèvement des biofilms de $16 \mathrm{~mm}$ de diamètre. La surface concave de ceux-ci est ajustée pour s'adapter à la forme du cylindre extérieur. Le cylindre interne est plein et entraîné par un moteur à vitesse réglable de façon à contrôler sa vitesse de rotation autour de son axe. La vitesse de rotation du cylindre interne détermine en partie le profil de cisaillement du fluide qui circule entre les deux cylindres. A l'entrée du réacteur, un divergent est installé pour assurer une distribution homogène du liquide entrant sur toute la hauteur du réacteur.

L'avantage de ce réacteur est de pouvoir assurer un écoulement Couette-Poiseuille qui n'est que le couplage d'un écoulement Couette avec un écoulement Poiseuille. Cet écoulement présente l'avantage de pouvoir travailler dans différentes configurations d'écoulement en variant le rapport vitesse de rotation du cylindre interne sur vitesse débitante du fluide [12].

Le profil de vitesse d'écoulement de Couette suivant le modèle de notre réacteur (cylindre interne mobile et cylindre externe fixe) est linéaire (figure 2). Ceci implique qu'une particule sera soumise au même gradient de vitesse $\left(\gamma_{p}\right)$ quelle que soit sa position dans le fluide, l'expression de la vitesse sera $\gamma_{p}=R_{\text {int }} \Omega /$ e avec $\Omega$ (radians) la vitesse de rotation du cylindre interne. Cette particularité de gradient de vitesse permet de calculer la force hydrodynamique à laquelle sera soumise une particule dans l'entrefer du réacteur. 

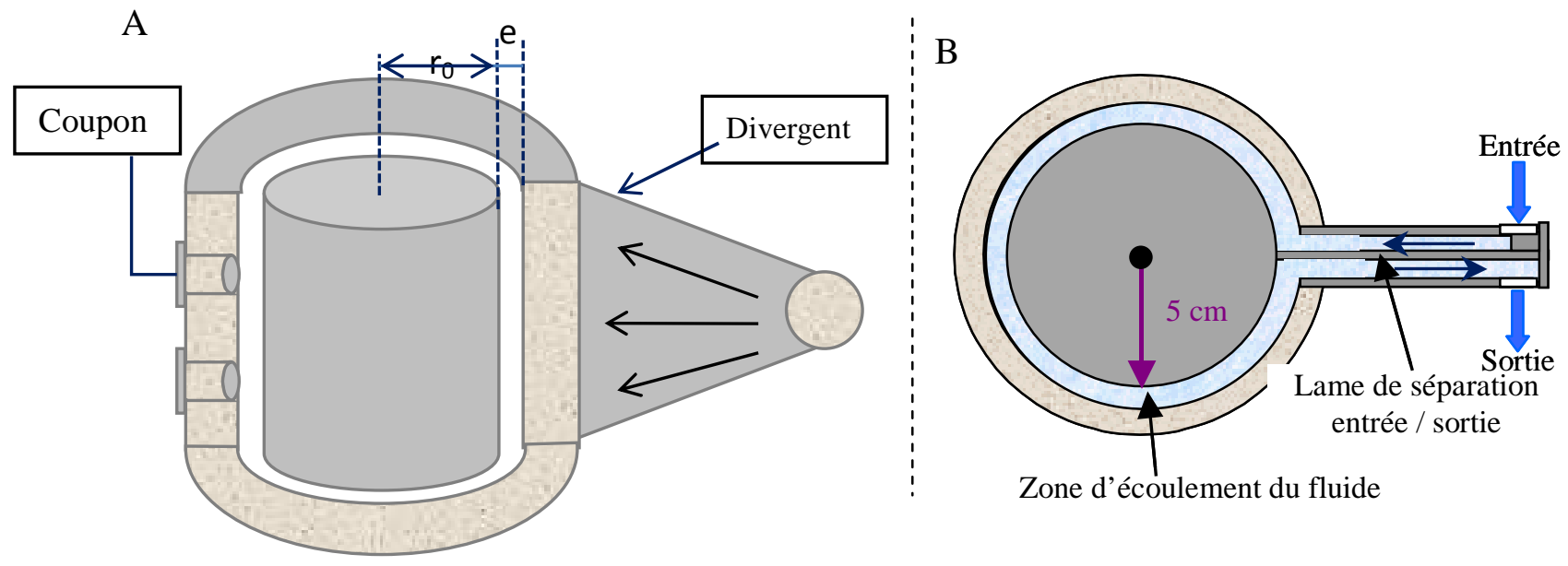

Figure1 : Coupe longitudinale (A) et transversale (B) du réacteur Couette-Poiseuille.

En ajoutant une entrée et une sortie à notre système (figure 1B), un écoulement de Poiseuille est obtenu. Dans le cas de nos conditions expérimentales, cylindre interne mobile, l'écoulement devient un écoulement Couette-Poiseuille. L'expression du gradient de cisaillement pour des petits espaces annulaires [12] est donnée par :

$$
\begin{gathered}
\gamma=R_{\text {int }} \Omega\left(1-6\left(\beta-\frac{1}{2}\right) \frac{1}{e}\right. \\
\beta=\frac{V_{q}}{R_{\text {int }} \Omega} \text { avec } \mathrm{V}_{\mathrm{q}} \text { la vitesse moyenne du fluide }
\end{gathered}
$$

Le facteur $\beta$ (rapport entre la vitesse débitante et la vitesse de rotation du cylindre interne) détermine le profil de vitesse et par la suite le gradient de cisaillement appliqué.

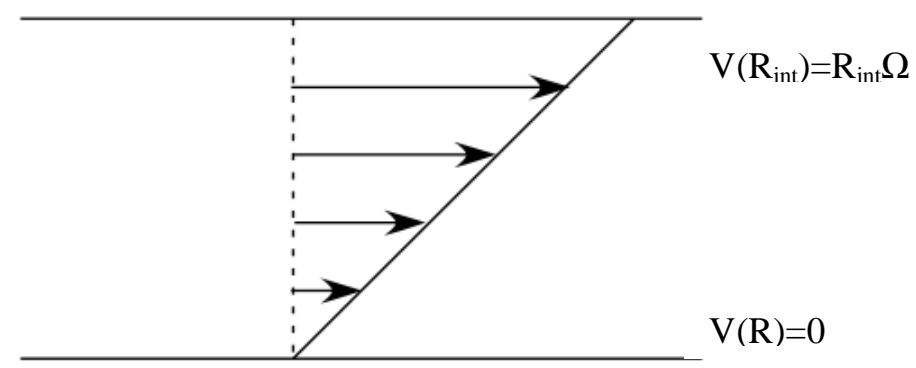

Figure2 : Profil de vitesse dans un écoulement Couette.

Dans nos expérience $\beta=1 / 2$, ce qui correspond à un écoulement de Couette strict (vitesse maximale d'écoulement à la surface du cylindre interne et nulle à la surface du cylindre externe). L'expression de gradient de cisaillement devient :

$$
\gamma=\frac{R \mathrm{int} \Omega}{e}
$$

\section{Mise en marche du réacteur}

15 litres d'eaux usées, collectées au niveau du bassin C de la STEP de Maxéville après le dessablage et le dégrillage des eaux usées, de façon à éviter les grosse particules et la graisse qui peuvent colmater la pompe, sont versées dans une cuve carrée en plexiglas qui sera le réservoir 
d'alimentation du réacteur. Cette eau est renouvelée quotidiennement pendant une semaine. L'eau usée dans la cuve est maintenue sous agitation à une vitesse de 150 tours/minutes à l'aide d'une palette repliable d'agitation en polypropylène (VWR 442-2121) L'eau est pompée dans un tuyau (Legris 1025406K02) en polyuréthane de 4mm de diamètre de la cuve à l'intérieur du réacteur à l'aide d'une pompe immergée à débit réglable (Fisher W75601) $\left(1 \mathrm{~s}^{-1}\right.$ et $\left.6 \mathrm{~s}^{-1}\right)$, (figure3). L'eau sortant du réacteur retourne ensuite à la cuve. La position du tuyau de retour joue un grand rôle dans le contrôle du débit de l'eau pompée, en effet le réglage du débit correspondant à la vitesse de cisaillementappliquée est réalisé par l'ajustement de la hauteur de ce tuyau.

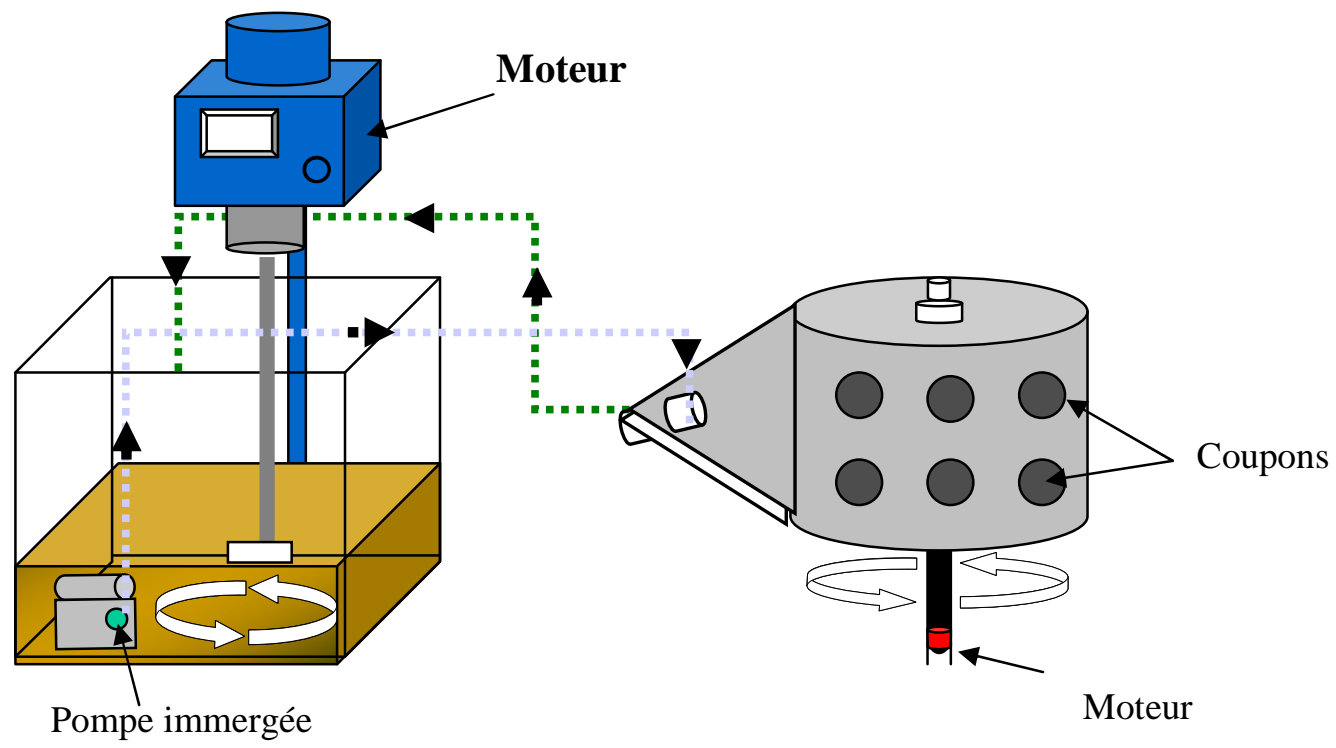

Figure 3 : Schéma du montage expérimental pour la croissance des biofilms.

Le suivie de l'évolution de l'eau usée est réalisé chaque heure par mesure du pH, conductivité, potentiel redox, oxygène dissous (WTW, Mutltiline F/SET) et turbidité (Hach 2100P). Un comptage bactérien sur la cellule de Thomas (VWR 720-0150) est effectué pour une évaluation du nombre de bactéries au début de la journée et au moment de renouvellement après $24 \mathrm{~h}$.

Un coupon, sur lequel se développe le biofilm, est prélevé chaque 24h, l'eau est éliminée en égouttant le coupon. Le coupon est ensuite pesé et placé dans une petite boite à bouchon en polyéthylène (partie contenant le biofilm vers le haut) contenant au fond quelque $\mathrm{mL}$ d'eau ultrapure. La boite est ensuite placée à $4^{\circ} \mathrm{C}$ avant l'observation en microscopie confocale à balayage laser. L'observation par microscopie confocale est réalisée le plus tôt possible (1 jour après le prélèvement des coupons) afin de minimiser une évolution possible du biofilm.

\section{Observation par microscopie confocale}

Avant d'observer les biofilms au CLSM, ces derniers sont colorés par l'acridine orange. Ce fluorochrome possède une longueur d'onde d'excitation et d'émission spécifique au marquage des acides nucléiques (ADN et ARN) ainsi que les exopolymères extracellulaires $[13,14]$. Pour réaliser cette coloration, à l'aide d'une micropipette $100 \mu \mathrm{L}$ d'acridine orange sont déposés sur l'échantillon de biofilm sur le coupon, le coupon est remué doucement pour disperser le colorant sur la totalité de la surface; le coupon est ensuite placé à l'obscurité pendant 20 à 30 min pour la 
fixation de l'acridine sur le biofilm. Pour éliminer l'excès de colorant, le coupon est lavé en ajoutant $100 \mu \mathrm{L}$ d'eau ultrapure à plusieurs reprises jusqu'à élimination de l'excès de l'acridine. Le coupon est ensuite placé sur un dispositif comprenant une lame en quartz, contenant de l'eau ultrapure pour assurer un atmosphère humide pour l'échantillon, et observé en microscopie confocale sous objectif $\mathrm{x} 4$ et $\times 20$ (figure 4). Les observations sont stockées sous forme de piles d'images (3D). Les piles d'images obtenues seront destinées à différents logiciels de traitements d'image en commençant par l'amélioration de la résolution et l'atténuation du bruit de fond, passant par la déconvolution 3D pour arriver à la fin à la reconstruction 3D et le calcul des différents paramètres (épaisseur du biofilm, dimension fractales, volume et co-localisation, etc...).

\section{Résultats et discussion}

Les expériences dans le réacteur de croissance sont effectuées sous deux forces de cisaillements $\left(1 \mathrm{~s}^{-1}\right.$ et $\left.6 \mathrm{~s}^{-1}\right)$. Ceci est réalisé dans le but de voir l'impact de la variation des forces hydrodynamiques sur la structure et la croissance du biofilm. Les algorithmes mathématiques appliqués par l'intermédiaire du logiciel Gocad sur les piles d'image 3D obtenues par microscopie confocale ont permis de calculer les épaisseurs des biofilms, la quantité de biomasse fixée ainsi que l'estimation du volume de polymères, la surface et le volume de biofilm qui occupe la surface de coupons.

Les résultats (figure 4) montrent que la formation du biofilm n'est pas similaire pour les deux gradients. Concernant l'épaisseur, les résultats montrent que les biofilms obtenus sous cisaillement $1 \mathrm{~s}^{-1}$ sont plus épais que ceux obtenus à $6 \mathrm{~s}^{-1}$. L'épaisseur parait augmenter avec le temps jusqu'à atteindre une valeur au-dessous de laquelle le biofilm se détache et son épaisseur décroit. Pour le cisaillement $1 \mathrm{~s}^{-1}$, une chute de l'épaisseur est observée après $72 \mathrm{~h}$ de mise en marche de réacteur puis elle re-augmente dans les jours qui suivent. Cette chute observée peut être due à un détachement de biofilm lors de sa croissance : le biofilm atteint une épaisseur très importante de sorte que l'espace entre les deux cylindres devient très étroit et les particules en suspension dans le fluide entrainent une partie du biofilm d'où une baisse de son épaisseur. A noter que les valeurs d'épaisseur obtenues sont probablement sous-estimées car une petite partie du biofilm se détache lors de la préparation des coupons pour l'observation en microscopie confocale.

Concernant les quantités de biomasse et de polymères produits, elles sont différentes dans les deux cas. A $6 \mathrm{~s}^{-1}$, la quantité de polymères et de bactéries dans la phase d'initiation à la formation de biofilm est beaucoup plus importante montrant que la croissance de biofilm est initialisé par la production de polymères qui vont assurer l'adhésion des bactéries. Une fois que le biofilm commence à croître, cette quantité de polymères devient proportionnelle à la quantité de bactéries et l'épaisseur du biofilm.

Pour le cisaillement de $1 \mathrm{~s}^{-1}$, le comportement est différent, la quantité de polymères et de bactéries augmentent avec la croissance du biofilm. Au dernier prélèvement, l'épaisseur du biofilm est maximale (environ 350 microns) et la quantité de biomasse est la plus élevée tandis que la quantité de polymères est très faible. Ceci peut être confirmé par les études qui trouvent une diminution de la production de polymères quand l'épaisseur du biofilm augmente $[3,11]$. Les résultats montrent aussi que la majorité des polymères élaborés à l'intérieur du biofilm semble former une matrice indépendante tandis que les bactéries semblent formées de deux catégories équivalentes une liées aux exopolymères et l'autre sans polymères. 

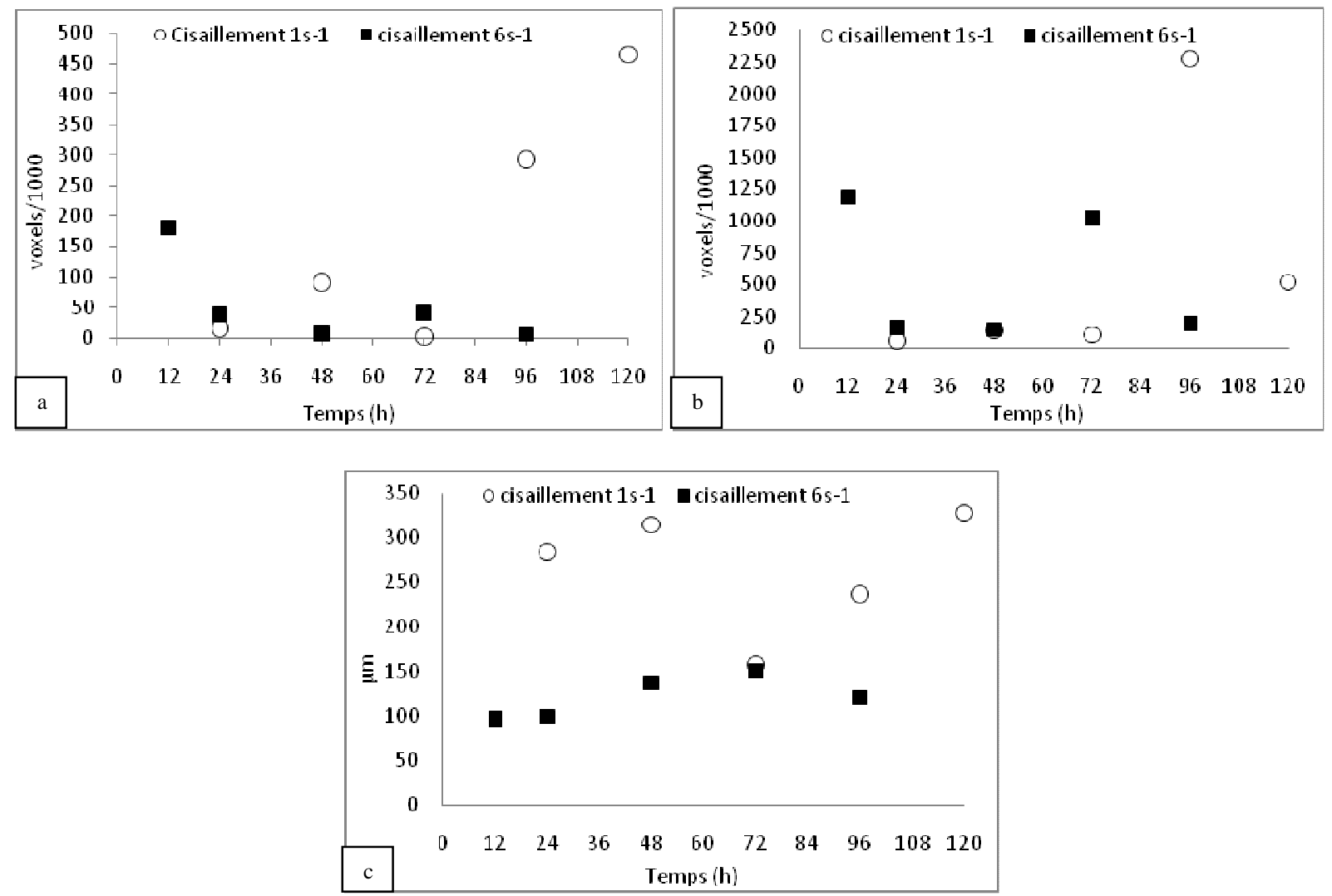

Figure 4 : Résultats obtenues à partir de Gocad : a) quantité de bactéries (exprimé par voxels), b) quantité de polymères (exprimé par voxels), c) épaisseur de biofilm sur le coupon

L'observation des coupons par microscopie confocale montre que pour les deux cisaillements, l'initiation au biofilm commence par l'adsorption de substrat sur le coupon suivi par l'adsorption des bactéries et le développement et l'agrégation de microcolonies. Pour le cisaillement $6 \mathrm{~s}^{-1}$ nous remarquons que la propagation des colonies bactériennes est surfacique, les colonies sont de petite taille mais occupent toute la surface du coupon. Pour le cisaillement $1 \mathrm{~s}^{-1}$, la prolifération des colonies se fait verticalement, le nombre de colonies occupant la surface des coupons est limité et la croissance se fait en épaisseur. Un phénomène commun observé sur tous les coupons est l'élongation des colonies dans le sens d'écoulement à un angle d'environ 45 degré. Ceci peut être corrélé aux forces de cisaillement qui entrainent les cellules filles lors de leur division dans le sens d'écoulement [7].

Deux expériences additionnelles, avec les mêmes gradients de cisaillement, en condition dit de «non dépôt » ont été réalisées dans le but d'avoir les conditions les plus défavorables pour la formation de biofilm. Dans ce cas, la vitesse d'écoulement est nulle à la surface du cylindre interne et maximale à la surface du cylindre externe. Les premiers résultats des observations en microscopie confocale montrent une différence nette entre les deux conditions. Que ce soit au gradient $6 \mathrm{~s}^{-1}$ ou $1 \mathrm{~s}^{-1}$ aucun développement de biofilm n'a été observé durant les deux premiers jours (figure 5). 

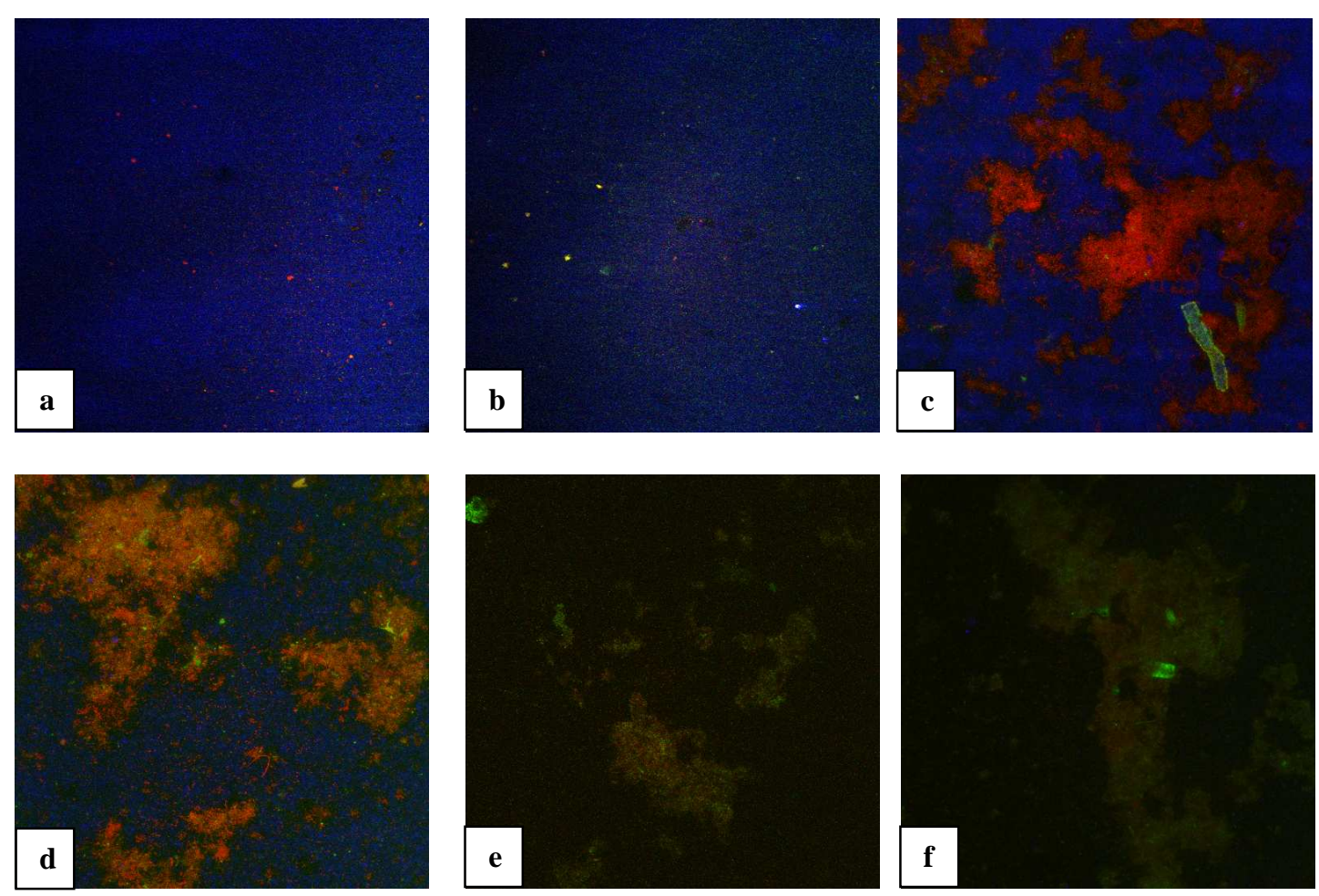

Figure 5 : Images obtenues par microscopie confocale (objectif x20) : a) coupon 24h condition non dépôt, b) coupon $48 \mathrm{~h}$ condition non dépôt, c et d) coupons respectivement 24 et $48 \mathrm{~h}$ (condition normale, cisaillement $6 \mathrm{~s}^{-1}$ ), e et f) coupons respectivement 24 et $48 \mathrm{~h}$ (condition normale, cisaillement $1 \mathrm{~s}^{-1}$ )

Après $48 \mathrm{~h}$, un dépôt de biofilm commence à se former dont l'origine est à rechercher sur les modifications des conditions hydrodynamiques sur la surface du cylindre interne du réacteur: en effet le nouveau profil de cisaillement, s'il défavorise la formation de biofilm sur les coupons, facilite la croissance de biofilm sur le cylindre interne.

\section{Conclusion}

Les expériences réalisées dans le réacteur Couette-Poiseuillle ont permis l'étude du développement des biofilms à partir des eaux usées dans un écoulement laminaire sous différents gradients de vitesses pariétales. La reconstruction 3D et l'application des algorithmes de calcul sur les données issus des images obtenues à partir de la microscopie confocale a permis d'étudier la structure et les caractéristiques des biofilms formés. Les biofilms présentaient deux modes différents de développement ainsi que des structures différentes selon le taux de cisaillement appliqué $\left(1 \mathrm{~s}^{-1}, 6 \mathrm{~s}^{-1}\right)$. La formation de biofilm se trouve très ralentie (au moins $48 \mathrm{~h}$ ) en appliquant les conditions de «non dépôt». Ceci s'avère très important pour toutes les applications nécessitant d'éviter le développement de biofilm. 


\section{Références}

1. Eberl H.J., P.C., Heijnen J.J, Van Loosdrecht M.C.M. 2000. A three-dimensional study on the correlation of spatial structure, hydrodynamic conditions, and mass transfer and conversion in biofilms. Chemical Engineering Sciences 55 p. 6209-6222.

2. Horn H., R.H., Morgenroth E.. 2003. Simulation of growth and detachement in biofilm systems under defined hydrodynamic conditions. Biotechnology and Bioengineering 81(5) p. 607-617.

3. Celmer D., O.J.A., Cicek N. 2008. Impact of shear force on the biofilm structure and performance of a membrane biofilm reactor for tertiary hydrogen-driven denitrification of municipal wastewater. Water Research 42 p. 3057-3065.

4. Andersson S., Characterization of bacterial biofilms for wastewater treatment. 2009, Royale Istitute of Technology, school of Biotechnology: Stocholm.

5. Abrahamson M., L.Z., Geesey G., Skjak-Braek G., Strand W., Christensen B.E.. 1996. Development of an artificial biofilm to study the effects of a single microcolony on mass transport. Journal of Microbiological Methods 26(1-2) p. 161-169.

6. Costerton.J.W.. 1999. Discussion : Introduction to biofilm. International Journal of Antimicrobial Agents 11 p. 217-221.

7. Stoodley P., D.I., Boyle J.D., Lappin-Scott H.M.. 1999. Influence of hydrodynamics and nutrients on biofilm structure. Journal of Applied Microbiology Symposium Supplement $85(28)$ p. 19S-28S.

8. Pereira M., K.M., Wuertz S., Neu T., Melo L.. 2002. Effect of flow regime on the architecture of a Pseudomonas fluorescens biofilm. Bitechnology and Bioengineering 78(2) p. 164-171.

9. Decho A.. 2000. Microbial biofilms in itertidal systems: An overview. Continental Shelf Research 20 p. 1257-1273.

10. Liu Y., T.J.-H.. 2002. The essential role of hydrodynamic force in the formation of biofilm and granular sludge. Water Research 36 p. 1653-1665.

11. Wagner M., I.N.P., Haisch C., Niessner R.. 2008. Combined use of confocal laser scanning microscopy (CLSM) and Raman microscopy (RM): Investigation on EPSMatrix. Water Research XXX p. 1-14.

12. Paris, T., Formation et organisation de biofilms en milieu eau potable. Influence du gradient de vitesse pariétale., in U.F.R Sciences \& Techniques de la Matière et des Procédés. Ecole Doctorale Energie Mécanique Matériaux, Département de Formation Doctorale Mécanique Energétique. 2008, Université Henri Point Carré: Nancy.

13. Wirtanen G., S.S., Helander I.M, Mattila-Sandholm T.. 2001. Microbiological methods for testing disinfectant efficiency on Pseudomonas biofilm. Colloids and Surfaces B: Biointerfaces 20(1) p. 37-50.

14. Jana-Sur P., C.A.K.. 2005. Studies on the interaction of bacterial capsular polysaccharide-Klebsiella K16 with cationic dyes. J. Photochem. Photobiol. Chem 173 p. 64-69. 